\title{
Perspectives on the Design of Clinical Trials Combining Transarterial Chemoembolization and Molecular Targeted Therapy
}

\author{
Chiun Hsu $^{\text {a,b }}$ Po-Ching-Liang ${ }^{c}$ Satoshi Morita ${ }^{d}$ \\ Fu-Chang Hu e Ann-Lii Cheng a,b,f \\ ${ }^{a}$ Department of Oncology, ${ }^{b}$ Department of Internal Medicine and 'Department of Medical Imaging, \\ National Taiwan University Hospital, Taiwan, ROC; dDepartment of Biostatistics and Epidemiology, \\ Yokohama City University Graduate School of Medicine, Yokohama, Japan; ${ }^{\mathrm{e} G r a d u a t e ~ I n s t i t u t e ~ o f ~}$ \\ Clinical Medicine and ${ }^{\mathrm{f}}$ Graduate Institute of Oncology, National Taiwan University College of \\ Medicine, Taiwan, ROC
}

\section{Key Words}

Clinical trial $\cdot$ Hepatocellular carcinoma $\cdot$ Transarterial chemoembolization

\begin{abstract}
Transarterial chemoembolization (TACE) moderately prolongs the survival of patients with intermediate-stage hepatocellular carcinoma. Molecular targeted therapy (MTT) may improve the efficacy of TACE. However, the findings of clinical trials evaluating the efficacy of a combination of TACE and MTT are conflicting. We hypothesized that this disparity can be prevented using alternative study designs. In this review, we classify the pertinent issues of study designs into five domains: primary endpoints, patients, TACE procedures, timing of randomization, and drug administration. Furthermore, we discuss the methods for increasing the success rate by minimizing potentially confounding factors within these five domains. Transarterial chemoembolization (TACE) is the current standard therapy for patients with Barcelona Clinic Liver Cancer (BCLC) intermediate-stage hepatocellular carcinoma (HCC) [1-3]. The survival benefit of TACE is supported by the results of meta-analysis of clinical trials comparing TACE with other conservative treatments in patients with inoperable HCC [4]. The results showed that the median survival of patients improved from approximately 16 to 20 months following TACE $[4,5]$. Although advances in TACE techniques and the use of new embolization agents may improve the efficacy of TACE $[6,7]$, other approaches are needed
\end{abstract}


to further improve the outcome in HCC patients treated using TACE. Molecular targeted therapy (MTT) has improved the survival of patients with advanced-stage HCC $[5,8]$. Therefore, combining MTT and TACE may additionally improve the survival in patients with intermediate-stage HCC. Many molecular targeted agents (MTA) are currently undergoing evaluation in randomized trials (table 1). However, the designs of these trials differ significantly. The results of two trials combining sorafenib and TACE were recently reported. Both trials failed to demonstrate a therapeutic benefit of the combination therapy for time to tumor progression (TTP) or overall survival (OS) $[9,10]$. However, specific subgroups of patients who received treatment for more than 6 months exhibited significantly better survival (table 2). Because median survival can be greater than 2 years in patients with intermediate-stage HCC, it is likely that an extended exposure period is necessary for MTA effects to reach the biological threshold at which survival benefit becomes measurable. Therefore, early discontinuation of study drug treatment may significantly undermine the statistical power of efficacy analysis in randomized trials (fig. 1). Clinical trials should be designed to minimize confounding factors that could lead to early discontinuation of study drug $[1-3,11,12]$. Factors that are crucial in this regard can be categorized into five domains: (1) selection of primary endpoints, (2) selection of patient population, (3) selection of TACE procedures, (4) timing of randomization, and (5) study drug administration. In this review we discuss the confounding effects potentially associated with each domain and the possible interactions among domains in trials combining TACE and MTA. We also discuss strategies that can help improve sensitivity and accuracy measurements of MTA efficacy.

Copyright (C) 2012 S. Karger AG, Basel

\section{Domain 1. Selection of Primary Endpoints}

Improvement in OS is the gold standard for measuring the efficacy of anticancer treatments. With regard to the testing of new drugs for advanced-stage HCC, randomized phase II trial designs that use TTP as the primary endpoint are recommended, in order to avoid the potential confounding effects of patients' underlying cirrhosis on OS [11]. However, this approach may be less reliable for patients with intermediate-stage HCC, for which OS benefit is the primary endpoint. In recent clinical trials of TACE for intermediate-stage HCC, the median TTP was found to be approximately 5-6 months. However, the median OS can exceed 2 years, indicating that most patients are exposed to the study drug for relatively short periods during clinical trials (fig. 1) [9, 10]. Unless the effect of the study drug can be sustained for a sufficient period of time, the OS benefit will be markedly attenuated.

In recent TACE trials, tumor progression was most often defined by either the Response Evaluation Criteria in Solid Tumors (RECIST) guidelines or the modified RECIST, which measure the size of viable tumors that are enhanced during the arterial phase of contrast-enhanced computed tomography or magnetic resonance imaging $[13,14]$. These definitions must be used carefully in TACE trials for two reasons. First, liver tumors, especially larger ones ( $>5 \mathrm{~cm}$ in diameter), usually undergo heterogeneous enhancement after TACE and response may be difficult to evaluate by measuring either the size or the extent of enhancement. Second, although the occurrence of new tumors in the liver after TACE initiation is considered as tumor progression, many physicians choose to continue TACE provided absolute contraindications are absent. Therefore, clinical trial protocols that are contradictory to the common practice of physicians will lead to early discontinuation of the study drug through reduced physician compliance. 


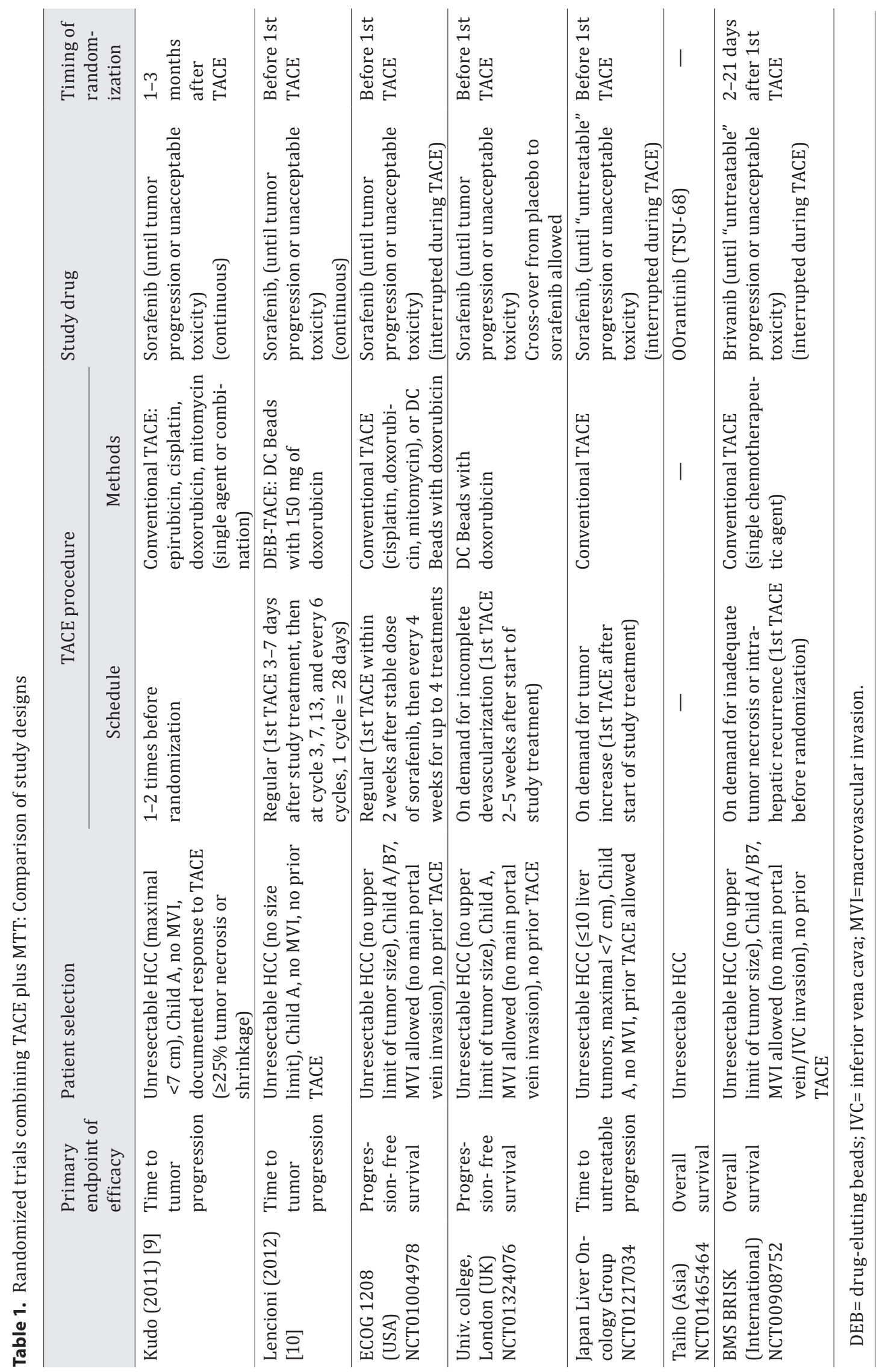


Because of these concerns, recent studies have led to the increasing use of time-to-untreatable progression or time-to-TACE failure as an endpoint in TACE trials $[3,15]$. The definition of untreatable progression can be categorized as follows: (1) absolute contraindication of TACE, including decompensated liver and kidney function, main portal vein thrombi, and untreatable arterioportal shunt; (2) expected poor response to further TACE, including continuing development of new hepatic tumors, despite adequately performed TACE and poor vascular access; (3) development of new vascular invasion or extrahepatic metastases; and (4) other relative contraindications of TACE. It appears that only categories (1) and (2) are unequivocally considered as untreatable progression. In other situations, physicians may decide to continue TACE if the major tumor burden remains in the liver. This may subsequently represent a potential confounding factor.

Furthermore, the definition of untreatable progression should not include subjective factors. For example, in the SPACE trial conducted by Lencioni et al. (2012), a reduction in performance status following TACE was considered as untreatable progression [10]. This was highly subjective, as proven by the dramatic difference between Asian and non-Asian patients who withdrew from the trial solely because of this factor. Therefore, a consensus on the definition of untreatable progression is crucial for TACE trials to ensure the homogeneity of study endpoints.

\section{Domain 2. Selection of Patient Population}

The inclusion of patients with tumors (e.g., maximum diameter greater than $10 \mathrm{~cm}$ ) or those with Child-Pugh class B cirrhosis requires further consideration. Such large tumors may respond poorly to TACE and progress rapidly following TACE. Larger tumors and ChildPugh B class cirrhosis may also predispose patients to TACE-related or study drug-related adverse events, especially in regard to liver decompensation. Therefore, the inclusion of these patients may increase the risk of early discontinuation of the study drug. Early study drug discontinuation may seriously undermine the statistical power of efficacy analysis, and greater sample sizes are needed to prove the efficacy of the study drug. It is thus critical to select the right patients to avoid early discontinuation of the study drug.

Most recent TACE trials targeted patients with BCLC intermediate-stage disease and some included those with tumor invasion of the portal vein branches. The efficacy of TACE in patients with vascular invasion is unclear. Most early TACE trials excluded patients with vascular invasion (table 1). In a Hong Kong trial that showed a survival benefit of TACE, a subgroup analysis in patients with vascular invasion $(n=21)$ revealed a nonsignificant difference in median survival (5.1 and 2.6 months in the TACE and control groups, respectively) [16]. However, in daily practice in most Asian countries, TACE is still an option of palliative therapy for patients with segmental portal vein invasion, and exclusion of these patients may reduce the accrual rate of TACE trials by $20-30 \%$.

\section{Domain 3. TACE Procedures}

The schedules and methods for TACE are heterogeneous. In clinical practice, TACE is usually performed once or twice to achieve tumor necrosis and further performed in cases of suspected residual or recurrent tumors, termed on-demand TACE [2, 3]. In most reported trials, TACE was performed at predefined schedules that were often every 2-3 months. In the context of clinical trials, scheduled TACE may avoid the potential imbalance of TACE treat- 
Fig. 1. Typical design of a clinical trial combining TACE and MTT. $\mathrm{ED}=$ early discontinuation ofstudy treatment before documentation of tumor progression or TACE failure.

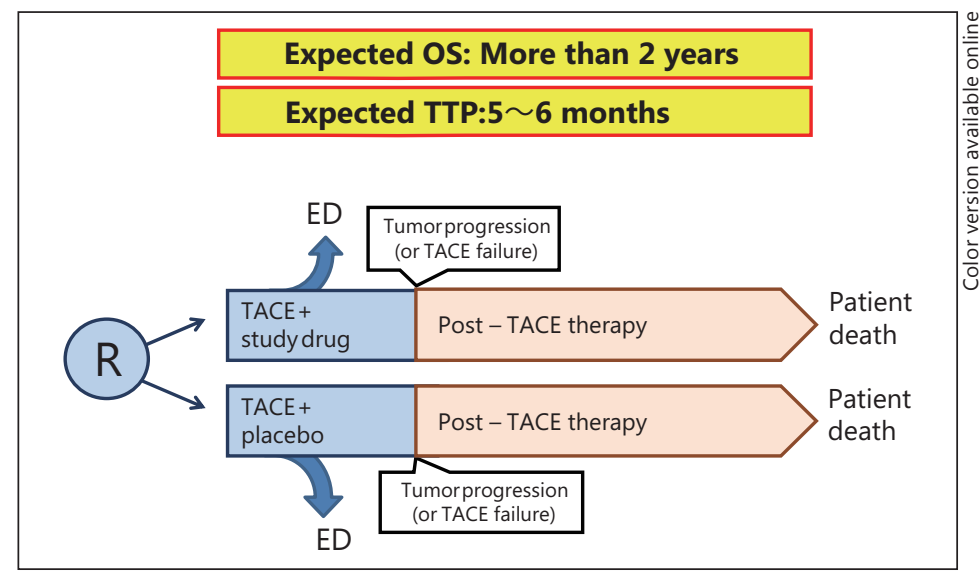

Table 2. Impact of treatment duration on clinical outcome: Examples from trials combining TACE plus sorafenib

\begin{tabular}{lcccc}
\hline & \multicolumn{2}{c}{ Japan-Korea trial [9] } & \multicolumn{2}{c}{ SPACE trial [10] } \\
\cline { 2 - 5 } & $\begin{array}{c}\text { Japan } \\
(\mathrm{n}=196)\end{array}$ & $\begin{array}{c}\text { Korea } \\
(\mathrm{n}=33)\end{array}$ & $\begin{array}{c}\text { Non-Asian } \\
(\mathrm{n}=200)\end{array}$ & $\begin{array}{c}\text { Asian } \\
(\mathrm{n}=104)\end{array}$ \\
\hline $\begin{array}{l}\text { Median duration of } \\
\text { study treatment* }\end{array}$ & 16 weeks & 31 weeks & 17.4 weeks & 30.0 weeks \\
\hline $\begin{array}{l}\text { TTP HR (95\% CI) } \\
\text { OS HR (95\% CI })\end{array}$ & $0.94(0.75-1.19)$ & $0.38(0.18-0.81)$ & $0.86(0.58-1.30)$ & $0.72(0.46-1.14)$ \\
\hline $\begin{array}{l}\text { Patients who } \\
\text { received TACE after } \\
\text { documented } \\
\text { progression }(\%)\end{array}$ & NA & NA & $1.06(0.65-1.74)$ & $0.68(0.36-1.29)$ \\
\hline
\end{tabular}

ment between treatment groups. However, scheduled TACE may be associated with an increased risk of treatment-related adverse events and liver decompensation $[3,17]$. Moreover, scheduled TACE may not reflect everyday practice for the majority of interventional radiologists and occasionally may not represent the best treatment option for patients. Therefore, scheduled TACE may increase the risk of early discontinuation of the study treatment and undermine the statistical power of efficacy analysis in TACE trials.

The optimal chemotherapeutic regimens used in conventional TACE remain undetermined. Recent research efforts have focused on the development of novel agents such as drugeluting beads. Results of a randomized TACE trial using doxorubicin-eluting beads indicated lower treatment-related toxicity and a trend of improved objective response compared with conventional doxorubicin treatment [7]. Although guidelines to standardize procedures using doxorubicin-eluting beads were recently established, little is known regarding the optimal dosing and scheduling of doxorubicin. Moreover, the principles of doxorubicin dose adjustment based on patients' liver function reserves and adverse events are unclear [18]. This implies that in a TACE trial using drug-eluting beads, the investigators will need some time to familiarize themselves with this new procedure. Without adequate experience of this novel delivery method, investigators may choose to withdraw subjects from study treatment and resort to conventional TACE when adverse events occur. The results of the study would thus 
be affected by the large number of patients undergoing further TACE after documented "untreatable progression" (table 2).

\section{Domain 4. Timing of Randomization}

An important rationale of combining TACE and MTA is to improve the outcome of TACE by inhibiting TACE-induced angiogenesis. Theoretically, to achieve this goal, antiangiogenic therapy should start before or immediately after TACE. A shortcoming of this approach is that it will enroll a significant proportion of patients who do not respond to or cannot tolerate TACE (up to $20 \%$ in recent TACE trials) $[7,10]$, thus increasing the risk of early discontinuation and nullifying the potential benefit of MTA. A second concern is that the response to TACE may not be balanced between the treatment and control groups. Furthermore, since subjects usually receive the study treatment immediately after randomization, the side effects of TACE and MTA may overlap and confound the interpretation of adverse events.

An alternative design is to randomize patients after achieving response to TACE, which can ensure a more homogenous and balanced patient population in terms of TACE response. However, this will delay the study treatment. A Japanese-Korean trial of sorafenib use following TACE reported a median duration of 9.3 weeks (range, 5.6-13.3 weeks) between final TACE and randomization [9]. This delay, which resulted mainly from the requirement for documented TACE response, precluded the possibility of inhibiting TACE-induced angiogenesis by sorafenib, an event that may occur within days after TACE $[19,20]$.

We suggest that the timing of randomization should be determined according to the mechanistic features of the MTA being tested. For antiangiogenic agents, randomization should be done before or immediately after initial TACE. Selection of the patient population should focus on patients with relatively low tumor burden and good expected response to TACE. Such patients would represent a more homogenous group, which can be maintained on the study drug for longer periods. For other MTA, randomization can be done after achieving a prespecified response to TACE. This can ensure homogeneity of the baseline patient characteristics and minimization of the risk of early discontinuation caused by tumor progression.

\section{Domain 5. Study Drug Administration}

To maximize the potential efficacy of MTA, a continuous treatment schedule was adopted in early TACE-based trials (table 1). However, the overlapping of side effects of TACE and MTA may have resulted in additional adverse events and early discontinuation of the study drug. A recent meta-analysis of clinical trials using vascular endothelial growth factor receptor kinase inhibitors in cancer patients indicated that this class of drugs is associated with increased risk of treatment-related death, with a relative risk of 2.23 (95\% confidence interval, 1.12-4.44) compared with control groups [21]. The most common causes of treatment-related death in these trials were bleeding, myocardial infarction, and liver dysfunction [21]. These adverse events were also the most commonly reported serious events in TACE-based trials. Although no evidence has established that a combination of TACE plus vascular growth factor receptor kinase inhibitors further increases the risk of bleeding, a trend toward increased liver toxicity was detected in two trials that used a combination of TACE and sorafenib $[9,10]$. 
Table 3. Toward better design of trials combining TACE plus MTT

\begin{tabular}{|c|c|}
\hline Key elements & Solutions \\
\hline Protocol harmonization & $\begin{array}{l}\text { - Define clearly the response criteria for TACE } \\
\text { - Define clearly "untreatable progression" pertinent to real-life clinical practice }\end{array}$ \\
\hline Patient selection & -Avoid patients with huge tumor burden and borderline liver function reserves \\
\hline Physician compliance & $\begin{array}{l}\text {-Adopt TACE regimens/schedules pertinent to real-life clinical practice } \\
\text { - Acknowledge the learning curves for new technology and new drugs }\end{array}$ \\
\hline
\end{tabular}

Although it is unclear whether the continuation of study drug during TACE is associated with early study drug discontinuation, recent TACE-based trials adopted an on-demand TACE schedule and an interrupted regimen of the study drug to avoid overlapping of side effects (table 1). The study drug was withheld temporarily, usually for several days to 2 weeks during each TACE session. Preliminary reports of these studies indicated good tolerance to the study drug $[22,23]$.

With regard to the new TACE techniques, investigators will need some time to familiarize themselves with the new study drugs, especially with regard to timely dose adjustment and better management of adverse events. These issues may have contributed to the early discontinuation of the study drug by Japanese and European investigators in both trials involving a combination of TACE and sorafenib (table 2). Recognition of this "learning curve" is a first step toward avoiding early discontinuation and ensuring homogeneity of study drugs.

\section{Summary: Is There An "Optimal” Design for Clinical Trials Combining TACE and MTA?}

The five domains above have complex interactions in the context of clinical trials combining TACE and MTA. To maximize the possibility of determining the potential efficacy of MTA, clinical trial designers should be mindful of protocol harmonization, patient selection, and physician compliance (table 3). In protocol harmonization, the definitions of response to TACE, demand for further TACE, and TACE failure (untreatable progression) should be defined clearly. We believe that definition of TACE failure should be restricted to patients with absolute contraindications for TACE (decompensated liver and kidney function, main portal vein thrombi, and untreatable arterioportal shunt) or to those with continually progressing intrahepatic tumors, despite adequately performed TACE. The treatment schedule should be designed to consider the potential overlapping toxicity of TACE and MTA. In patient selection, especially in early proof of concept trials, patient populations with borderline liver function reserves or a large tumor burden should be avoided. These steps will help reduce early discontinuation of the study drug and increase the number of opportunities to detect the potential efficacy of the MTA. For enhanced physician compliance, the trial design should be pertinent to actual clinical practice to ensure TACE protocol adherence and to reduce the heterogeneity of post-TACE therapy. This will be especially important when OS is used as the primary endpoint. 


\section{Conflict of Interest}

Dr. Ann-Lii Cheng is a consultant for Sanofi-Aventis Inc, Pfizer, Bayer Schering Pharma, Bristol-Myers Squibb (Taiwan) Ltd, Boehringer Ingelheim Taiwan Limited, and Novartis Inc. Dr. Chiun Hsu is a member of the speakers' bureau of Bayer-Schering Pharma. Other authors have no relevant financial interests related to this article.

\section{References}

1 Bruix J, Sherman M: Management of hepatocellular carcinoma: an update. Hepatology 2011;53:10201022.

2 Han KH, Kudo M, Ye SL, Choi JY, Poon RT, Seong J, et al: Asian consensus workshop report: expert consensus guideline for the management of intermediate and advanced hepatocellular carcinoma in Asia. Oncology 2011;81(suppl 1):158-164.

3 Raoul JL, Sangro B, Forner A, Mazzaferro V, Piscaglia F, Bolondi L, et al: Evolving strategies for the management of intermediate-stage hepatocellular carcinoma: available evidence and expert opinion on the use of transarterial chemoembolization. Cancer Treat Rev 2011;37:212-220.

4 Llovet JM, Bruix J: Systematic review of randomized trials for unresectable hepatocellular carcinoma: chemoembolization improves survival. Hepatology 2003;37:429-442.

5 Llovet JM, Bruix J: Novel advancements in the management of hepatocellular carcinoma in 2008. J Hepatol 2008;48:S20-S37.

6 Takayasu K, Arii S, Kudo M, Ichida T, Matsui O, Isumi N, et al: Superselective transarterial chemoembolization for hepatocellular carcinoma. Validation of treatment algorithm proposed by Japanese guidelines. J Hepatol 2012;56:886-892.

7 Lammer J, Malagari K, Vogl T, et al: Prospective randomized study of doxorubicin-eluting- bead embolization in the treatment of hepatocellular carcinoma: results of the PRECISION V study. Cardiovasc Intervent Radiol 2010;33:41-52.

8 Hsu C, Shen YC, Cheng AL: Anti-angiogenic strategies for the treatment of hepatocellular carcinoma. In Bolondi L (eds) Targeted therapy for hepatocellular carcinoma. pp 18-32. Future Science Group E-Books. Future Science Group, United Kingdom, 2011 (doi: 10.2217/ebo.11.170).

9 Kudo M, Imanaka K, Chida N, Nakachi K, Tak WY, Takayama T, et al: Phase III study of sorafenib after transarterial chemoembolisation in Japanese and Korean patients with unresectable hepatocellular carcinoma. Eur J Cancer 2011;47:2117-2127.

10 Lencioni R, Llovet JM, Han G, Tak WY, Yang J, Leberre MA, et al: Sorafenib or placebo in combination with transarterial chemoembolization (TACE) with doxorubicin-eluting beads (DEBDOX) for intermediatestage hepatocellular carcinoma (HCC): Phase II, randomized, double-blind SPACE trial. J Clin Oncol 2012; 30 (suppl 4; abstr LBA154).

11 Llovet JM, Di Bisceglie AM, Bruix J, Kramer BS, Lencioni R, Zhu AX, et al: Design and endpoints of clinical trials in hepatocellular carcinoma. J Natl Cancer Inst 2008;100:698-711.

12 Strebel BM, Dufour JF: Combined approach to hepatocellular carcinoma: a new treatment concept for nonresectable disease. Expert Rev Anticancer Ther 2008;8:1743-1749.

13 Lencioni R, Llovet JM: Modified RECIST (mRECIST) assessment for hepatocellular carcinoma. Semin Liver Dis 2010;30:52-60.

14 Riaz A, Miller FH, Kulik LM, Nikolaidis P, Yaghmai V, Lewandowski RJ, et al: Imaging response in the primary index lesion and clinical outcomes following transarterial locoregional therapy for hepatocellular carcinoma. JAMA 2010;303:1062-1069.

15 Kudo M: Hepatocellular carcinoma in 2011 and beyond: from the pathogenesis to molecular targeted therapy. Oncology 2011;81(suppl 1):1-10.

16 Lo CM, Ngan H, Tso WK, Liu CL, Lam CM, Poon RT, et al: Randomized controlled trial of transarterial lipiodol chemoembolization for unresectable hepatocellular carcinoma. Hepatology 2002;35:1164-1171.

17 Makuuchi M, Kokudo N, Arii S, Futagawa S, Kaneko S, Kawasaki S, et al: Development of evidence-based clinical guidelines for the diagnosis and treatment of hepatocellular carcinoma in Japan. Hepatol Res 2008;38:37-51.

18 Lencioni R, de Baere T, Burrel M, Caridi JG, Lammer J, Malagari K, et al: Transcatheter treatment of hepatocellular carcinoma with doxorubicin-loaded DC bead (DEBDOX): technical recommendations. Cardiovasc Intervent Radiol 2011 Oct 19. [Epub ahead of print].

19 Sergio A, Cristofori C, Cardin R, et al: Transcatheter arterial chemoembolization (TACE) in hepatocellular carcinoma (HCC): the role of angiogenesis and invasiveness. Am J Gastroenterol 2008;103:914-921.

20 Shim JH, Park JW, Kim JH, et al: Association between increment of serum VEGF level and prognosis after transcatheter arterial chemoembolization in hepatocellular carcinoma patients. Cancer Sci 2008;99:2037-2044.

21 Schutz FA, Je Y, Richards CJ, Choueiri TK: Meta-analysis of randomized controlled trials for the incidence and risk of treatment-related mortality in patients with cancer treated with vascular endothelial growth factor tyrosine kinase inhibitors. J Clin Oncol 2012;30:871-877. 
DOI: $10.1159 / 000343830$

Published online: November 26, 2012

(c) 2012 S. Karger AG, Basel www.karger.com/lic

Hsu et al.: Design of Clinical Trials Combining TACE and MTT

22 Arai Y, Inaba Y, Yamamoto T, Kanai F, Aramaki T, Tanaka T, et al: A randomized phase II study of TSU-68 in patients (pts) with hepatocellular carcinoma (HCC) treated by transarterial chemoembolization. J Clin Oncol 2010; 28:15s (suppl; abstr 4030).

23 Chung Y, Kim B, Chen C, Wang J, Chu H, Yoon J, et al: Study in Asia of the combination of transcatheter arterial chemoembolization (TACE) with sorafenib in patients with hepatocellular carcinoma (HCC) trial (START): second interim safety and efficacy analysis. J Clin Oncol 2010; 28:15s (suppl; abstr 4026). 